DOI: https://doi.org/10.11144/Javeriana.upsy20.pcac

\title{
Prevalencia y características del acoso y ciberacoso entre adolescentes*
}

\section{Prevalence $\&$ Characteristics of Bullying $\&$ Cyberbullying among} Adolescents

\author{
Miguel Jiménez SÁnCHeZ \\ Universidad de Granada, España \\ ORCID: https://orcid.org/0000-0002-3704-917X \\ Emilio BerRocal de Luna ${ }^{a}$ \\ Universidad de Granada, España \\ ORCID: https://orcid.org/0000-0002-3562-8135 \\ Marta Alonso Ferres \\ Universidad de Granada, España \\ ORCID: https://orcid.org/0000-0002-2624-1131
}

a Autor de correspondencia. Correo electrónico: emiliobl@ugr.es

Para citar este artículo: Jiménez Sánchez, M., Berrocal de Luna, E., \& Alonso Ferres, M. (2021). Prevalencia y características del acoso y ciberacoso entre adolescentes. Universitas Psychologica, 20, 1-14. https://doi.org/10.11144/Javeriana.upsy20.pcac

\section{RESUMEN}

En el presente artículo, se analizan experiencias de acoso y ciberacoso con el propósito de conocer tanto las características que las envuelven como su prevalencia. Participaron 220 estudiantes desde sexto de educación primaria a primero de bachillerato, escolarizados en centros educativos de la provincia de Granada, seleccionados mediante un muestreo incidental no probabilístico. Para el diseño de la investigación, se optó por un enfoque metodológico cuantitativo no experimental, amparado bajo la técnica de encuesta, utilizando una versión adaptada del cuestionario Ciberbull, utilizado por Giménez et al. (2015). Los resultados mostraron una propensión elevada en ambas manifestaciones de acoso (23.6\% acosadores y $14.1 \%$ victimas), detectando que estas situaciones tendían a disminuir conforme al aumento de la edad del alumnado. Además, se hallaron diferencias, por un lado, en el sexode los sujetos que perpetran acoso $\left(M_{\text {hombres }}=1.2 ; D E=0.4\right.$ vs. $\left.M_{\text {mujeres }}=1.09 ; D E=0.29\right) \mathrm{y}$, por otra parte, en la prevalencia de victimización $(\beta=0.542, p<0.05)$, poniéndose de manifiesto que el hecho de ser acosado, puede ser un valor predictivo de ser víctima de ciberacoso. Por último, se recomienda continuar el estudio, realizando un análisis factorial exploratorio del instrumento, así como ampliando la muestra de participantes.

\section{Palabras clave}

acoso escolar; adolescencia; ciberacoso; prevalencia; sexo.

\section{ABSTRACT}

In this article we analyse experiences of bullying and cyberbullying to know the characteristics that surround them and their prevalence. The participants were 220 students from the sixth year of primary education to first year of high school education schooled in the province of Granada, selected through an incidental or non-probabilistic sampling. For the design of the research, a non-experimental quantitative methodological approach, supported under the survey technique, was chosen using an adapted version of the Ciberbull questionnaire by Giménez et al. (2015). The results showed a high propensity in both manifestations of bullying 
(23.6\% aggressors and $14.1 \%$ victims), detecting that these situations tended to decrease as the students' age increases. In addition, differences were found, on the one hand, in the gender of the perpetrators of bullying $\left(\mathrm{M}_{\mathrm{boy}}=1.2 ; \mathrm{SD}=\right.$ 0.4 versus $\left.M_{\text {girl }}=1.09 ; S D=0,29\right)$ and, on the other hand, in the prevalence of victimization $(\beta=0.0542, p<0.05)$, showing that being harassed may be a predictive value of being a cyberbullying victim. Finally, it is recommended to continue the study, carrying out an exploratory factor analysis of the instrument, as well as expanding the sample of participants.

Keywords

bullying; adolescence; cyberbullying; prevalence; gender.

El acoso (bullying) es definido por Olweus (1993, 2013) como 'un fenómeno que se produce cuando alguien más débil, el cual no puede defenderse, se halla expuesto de forma continuada, y durante un prolongado periodo de tiempo, a los comportamientos negativos de una o más personas'. Asimismo, se caracteriza por la intencionalidad, la repetición y un desequilibrio sistemático de poder y dominación evidente entre la víctima y el autor o autores que perpetran la agresión.

Esta temática ha suscitado el interés de la comunidad científica durante los últimos años (Cañón-Rodríguez, 2018; Castañeda-Vázquez et al., 2020; Herrera-López et al., 2018) y, aunque algunos estudios sobre su prevalencia han señalado que en cierto grado va decreciendo (Gázquez et al., 2010; Slonje et al., 2012), sigue siendo un problema preocupante que afecta a un gran número de escolares. Esta realidad se refleja, por ejemplo, en estudios como el de Nacimiento y Mora-Merchán (2014), donde se advierte que, de una muestra compuesta por 346 sujetos de edades comprendidas entre 12 y 18 años, un 47.2 \% había sufrido acoso ocasional (hasta dos veces al mes), mientras que un $40.6 \%$ lo padecía al menos una vez a la semana. En la misma línea se encuentra el estudio de Giménez et al. (2014), que señala que un $12 \%$ de una muestra de 1353 adolescentes españoles informó soportar experiencias de acoso escolar. O el de Cerezo y Alto (2010), donde se señala que un $17.3 \%$ de los adolescentes sufre acoso, encontrándose más agresores (8.6\%) que víctimas (8.3\%).
Las manifestaciones de acoso pueden ser clasificadas en torno a dos patrones: directo e indirecto (Powell \& Ladd, 2010). Los primeros estudios concernientes a esta modalidad de acoso escolar giraban en torno a sus implicaciones más directas, y es que el acoso directo puede subdividirse a su vez en dos: físico y verbal (Calmaestra, 2011). A su vez, la forma más indirecta hace alusión al maltrato psicológico (manipulación de relaciones, expansión de rumores) y la exclusión social (Ortega \& Monks, 2007).

A la hora de abordar investigaciones de esta índole, son diversas las variables que trascienden en ellas. Por ejemplo, la edad ha sido tenida en cuenta para algunos autores como Piñuel y Oñate (2007), quienes señalan que en el intervalo de edad 10-12 años, la incidencia de casos de acoso escolar es alta. Sin embargo, otros autores como Fitzpatrick et al. (2007), distinguen que la mayor etapa de prevalencia se sitúa en torno a los 14 años, inmiscuyéndose de esta manera dentro de la educación secundaria. Pero parece ser que la tendencia más común es que el número de víctimas disminuya de una etapa a otra, es decir, se reduce conforme aumenta la edad (Olweus, 2013). Posiblemente, la respuesta a este descenso pueda explicarse en el mejor uso que el alumnado de más edad hace de las estrategias de afrontamiento para responder a tales abusos, en comparación con los más pequeños. Otro factor que bien puede incidir en este descenso es el clima instaurado en el aula (López et al., 2012). A colación de lo mencionado, entre la estrategia de afrontamiento más utilizada por los escolares para paliar su implicación en situaciones de acoso, según se halla en el estudio de Naylor et al. (2001), es informar a otros sobre su situación. Comunicar el acoso también es una de las estrategias más empleadas por los escolares, en investigaciones como la llevada a cabo por De la Caba y López (2013). Desde otra perspectiva, el estudio de Polo et al. (2017) constata que la aplicación de técnicas de aprendizaje cooperativo reduce considerablemente las dinámicas de acoso.

En la literatura existente al respecto, también se muestra que la variable sexo está asociada a la 
prevalencia en estudios acerca del acoso (Ortega et al., 2008). Los hombres tienden a estar más implicados en comparación con las mujeres en los casos de acoso directo, tanto en el rol de agresores, como de víctimas. No obstante, las investigaciones sugieren que, en la ocurrencia de acoso indirecto, son esta vez las niñas quienes se encuentran más involucradas respecto a los niños (Scheithauer, 2002). Si bien, hay otros autores en cuyas investigaciones no se perciben diferencias significativas en cuanto al sexo, como por ejemplo, el trabajo de Campbell et al. (2012).

Por otra parte, el vertiginoso avance de las tecnologías ha otorgado a los menores nuevas formas de comunicarse, pero estas no están siempre bien empleadas, lo que genera dinámicas sociales nocivas (Kowalski \& Limber, 2007) que han dado lugar a la aparición de un progresivo fenómeno de intimidación entre los jóvenes, conocido como acoso cibernético o ciberacoso, instaurándose como una nueva forma de violencia escolar.

En la actualidad, el ciberacoso es un hecho que va in crescendo como así se afirma en el estudio de Modecki et al. (2014). Al contrario del acoso, el acoso cibernético no cuenta con una definición consensuada, la principal causa se halla en la disensión de pareceres surgidos en torno a los criterios de repetición y desequilibrio de poder (Slonje et al., 2013), aunque con frecuencia, sí existe consonancia en reseñarlo como un proceder agresivo e intencionado que sucede a través de las TIC (Tecnologías de la Información y la Comunicación) (Francisco et al., 2015). Algunas de las particulares que caracterizan al ciberacoso es que para que este se produzca, en el momento en el que se lleva a cabo el ataque, debe haber menores en ambos extremos, si en alguno de ellos se encuentra un adulto, no podría considerase como tal. En ese caso, derivaría en otros tipos de acoso cibernético como por ejemplo el grooming, la sextorsión, etc. (Garaigordobil, 2011).

Los trabajos realizados en lo referente al acoso escolar, ya sea tanto por la vía tradicional, como por vía online, no son suficientes para ratificar las tasas de prevalencia en torno a esta temática, si bien la certeza hallada exhorta a proseguir investigando ante la evidencia de que un considerable porcentaje de estudiantes ha experimentado este fenómeno, y las perniciosas consecuencias que consigo asocia. En esta línea, las investigaciones hasta la fecha reportan resultados dispares en cuanto a las tasas de prevalencia.

Por ejemplo, en lo que respecta en este caso al ciberacoso, el trabajo de Estévez et al. (2010), reporta que un $30.1 \%$, de la muestra conformada por 1431 adolescentes con edades que oscilan entre 13 y 17 años, confesaron haber padecido algún tipo de ciberataque. Por su parte Polo et al. (2014), con una muestra de 620 escolares, correspondiente a los cursos de $5 . \mathrm{o}$

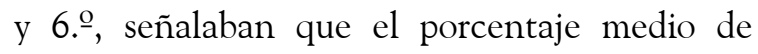
agresores online se situaba en el $5.4 \%$, mientras que el de cibervíctimas era del $8.7 \%$.

Por otro lado, según Tokunaga (2010), la prevalencia del ciberacoso varía en función de la edad y el nivel educativo, este autor sugiere que el periodo donde más ciberbulling se produce es en la adolescencia, arguyendo para ello la existencia de una relación curvilínea entre la edad y la victimización, situándose el punto más álgido de incidencia entre los 13 y 15 años.

En cuanto a la variable sexo, la mayoría de las investigaciones sitúan a las mujeres en el papel de víctimas (Giménez et al., 2014; Schneider et al., 2012). No obstante, otros autores no han encontrado desigualdades significativas en cuanto a sexo se refiere, como son el caso de Juvonen y Gross (2008) y Álvarez-García et al. (2011), entre otros.

A tal diversidad se une la variedad de metodologías e instrumentos utilizados (Modecki et al., 2014) que hacen que el comparar resultados no sea una tarea fácil, es decir, distintos instrumentos empleados para medir la prevalencia, conlleva a la consecución de resultados complejos.

Por ello, ante estas situaciones de acoso y ciberacoso, basándose en lo expuesto y a la luz de los diversos estudios llevados a cabo en territorio nacional y, sobre todo, los realizados en la Comunidad Autónoma de Andalucía como son el caso, por ejemplo, de Calmaestra (2011) y Nacimiento y Mora-Merchán (2014), 
se plantea la siguiente cuestión: ¿Cuáles son las situaciones de acoso y ciberacoso que actualmente pueden llegar a acontecer en el alumnado de la provincia de Granada? Dado que apenas existen estudios sobre dicha población, se precisa focalizar la atención del presente trabajo sobre los mencionados jóvenes. Con base en lo mencionado, este trabajo persigue dos objetivos principales: 1) identificar la prevalencia de acoso y ciberacoso entre adolescentes y 2) conocer las características de acoso y ciberacoso en función del rol (víctima/agresor).

\section{Método}

La metodología utilizada es cuantitativa no experimental, amparada bajo la técnica de encuesta. En este sentido, se realizaron dos tipos de diseños: una primera parte se basó en el método descriptivo para tratar de dar respuesta a los objetivos planteados, y es que haciendo alusión a Colás (1994), el principal propósito de los métodos descriptivos es "describir sistemáticamente hechos y características de una población dada o área de interés de forma objetiva y comprobable" p. 177. Asimismo, una segunda parte se sustentó en el método comparativo con la finalidad de verificar las hipótesis que también se establecieron.

\section{Hipótesis}

Delimitados los objetivos, las hipótesis que se plantearon para este estudio, y las hipótesis alternativas que surgieron de ellas, fueron las siguientes:

$\mathrm{H}_{1}$. Existe una menor prevalencia de acoso en la medida que el alumnado tiene una mayor edad.

$\mathrm{H}_{1.1}$. Existen diferencias significativas en el acoso en función del curso.

$\mathrm{H}_{2}$. La prevalencia de acosadores/agresores es mayor en los alumnos que en las alumnas.

$\mathrm{H}_{3}$. Existen diferencias en la prevalencia de cibervictimización en función si previamente se ha sido acosado/a.
$\mathrm{H}_{3.1}$. El hecho de sufrir acoso es un predictor de ser ciberacosado.

Muestra

En el estudio, participaron 220 estudiantes (53.6\% mujeres, $43.4 \%$ hombres), con una media de edad de 13.84 años, de centros educativos públicos desde sexto de educación primaria hasta primero de bachillerato (18.6 $\%, 17.3 \%, 27.7 \%, 8.6 \%, 18.6 \%$ у $9.1 \%$, respectivamente) escolarizados en la provincia de Granada, que fueron seleccionados mediante un muestreo incidental. Si bien es cierto que para poder aplicar el instrumento de recogida de información en los tres centros educativos seleccionados se requirió de la autorización de la Delegación Territorial de la Consejería de Educación en Granada, se contaba con la precedente aprobación de los directores, lo cual es bastante significativo, puesto que no todos los centros a los que se intentó acceder mostraron la misma disponibilidad a participar en estudios de esta índole.

\section{Instrumento}

El instrumento utilizado para la recogida de datos en el presente estudio fue una adaptación del Cuestionario de Autoinforme Ciberbull, utilizado en Giménez et al. (2015). Este instrumento parte de la revisión de otros utilizados en la investigación del acoso y el ciberacoso, llevados a cabo en territorio nacional (Calmaestra, 2011; García-Fernández et al., 2013), así como de otros cuestionarios de temática similar y contenido afín.

En su origen, consta de veintiséis cuestiones de carácter cerrado y abierto, con varios modelos de respuesta, donde se hallan las de tipo dicotómico (Sí/No), empleadas para detectar los posibles casos de acoso y ciberacoso; las de opción múltiple y de escala tipo Likert, que cuentan con cuatro o cinco opciones de respuesta en función de la pregunta, en las que se utilizan, bien sea cuantificadores lingüísticos de frecuencia, escalas 
de frecuencia o de duración, con la finalidad de medir los posibles casos de acoso por ambas vías.

Por otro lado, dado que los escolares a los que se les aplicó la adaptación surgida se hallaban en los mismos cursos a los que se dirigía el cuestionario Ciberbull en su origen

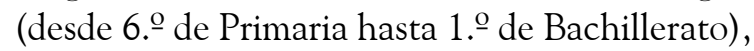
las modificaciones no fueron excesivas, y los contextos de la región de Murcia presentan características similares a las que pueden hallarse en la provincia de Granada, no se consideró necesario someter la adaptación resultante al proceso de fiabilidad, limitando nuestra intervención a otros aspectos técnicos como la claridad y precisión de los ítems y la validez de contenido. La claridad y precisión de los ítems se realizó con jueces conocedores del contexto, quienes evaluaron los ítems, procediendo a eliminar los considerados como no claros o innecesarios. En referencia a la validez de contenido, se fue fiel a las publicaciones habidas sobre el tema y se comprobó que el cuestionario utilizado era fiel reflejo de la teoría existente.

Explicado esto, y basándose en la validez de las inferencias, se siguieron las aportaciones de autores como Gómez e Hidalgo (2002) quienes sostienen que el concepto de validez ha ido transformándose en el transcurso del tiempo, señalando que esta viene definida como el grado en el que instrumento, como es el cuestionario en este caso, mide lo que se desea medir, es decir, lo validado no es el cuestionario en sí, sino la interpretación que se haga de los datos obtenidos por un procedimiento previamente establecido. En este sentido, Messick (1989) enuncia que la validez no se realiza sobre el cuestionario, sino que lo que realmente se valida son las inferencias que dimanan de los resultados, hallando en este camino las consecuencias sociales y éticas que este pudiera llegar a producir.

\section{Procedimiento de recogida de datos}

En primer lugar, se contactó con dos directores y un vicedirector de los centros, quienes posteriormente se prestaron a participar en este estudio, donde se les explicó la investigación. Una vez obtenido su visto bueno, se procedió a solicitar la autorización de la Delegación Territorial de la Consejería de Educación en Granada. Una vez que se contó con este permiso, se concertó otra cita con las figuras representativas de los centros mencionadas anteriormente para proporcionarles los cuestionarios a cumplimentar por el alumnado, que fueron distribuidos a los tutores de los cursos seleccionados, quienes los pasaron en horario de tutoría. Este modo de proceder se debió a que en las fechas elegidas el alumnado se encontraba en época de exámenes y no se quería interrumpir la agenda de los centros. Tras una semana de plazo, se volvió a concretar una cita para la recogida de los datos aportados en los respectivos cuestionarios.

\section{Análisis de datos}

Para el tratamiento de los datos obtenidos a través de los cuestionarios, se utilizó el programa estadístico SPSS en su versión 22. En una primera instancia, se optó por un análisis descriptivo de las variables que intervienen en cada uno de los objetivos para, además de darles respuesta, obtener un conocimiento más profundo del contexto, de forma que permitiera realizar interpretaciones más precisas y pulcras.

Posteriormente, y tras obtener una distribución normal de la muestra según la prueba K-S, se realizó una serie de comparaciones, efectuando para ello diversas pruebas paramétricas ajustadas a la necesidad de cada hipótesis de partida. Concretamente, se ejecutaron: correlaciones bivariadas mediante el coeficiente de correlación de Pearson; contraste de medias (pruebas $t$ Student); un ANOVA de un factor con un posterior análisis post-hoc y una regresión lineal, con el fin de poner a prueba las hipótesis de modo que se facultara la opción de verificarlas o no. 


\section{Resultados}

\section{Estadísticos descriptivos}

Para tratar de dar respuesta a los objetivos propuestos, en primer lugar, de forma global, se comprobó la cantidad de alumnos y alumnas que se encontraban implicados en situaciones de acoso y ciberacoso, diferenciando, por un lado, a víctimas y cibervíctimas y, por otro, a agresores y ciberagresores, distinguiendo seguidamente por sexo y cursos al alumnado vinculado con este fenómeno. Asimismo, se ahondó en las características de este, en las cuales se abarcó desde el tiempo que eran acosados/ciberacosados y el tiempo desde el que se cometía el acoso/ ciberacoso, la frecuencia, qué o sobre qué persona intervenían, cómo y también se registró si ese maltrato se contaba a otras personas, ya fuera como víctima/cibervíctima o como agresor/ ciberagresor.

Un $26.3 \%$ del total de la muestra de estudiantes seleccionada afirmaron ser o haber sido acosados en algún periodo de tiempo durante su estancia en el centro escolar. Por su parte, un $14.1 \%$ señaló haberse involucrado en el rol de agresor. En referencia al ciberacoso, un $13.2 \%$ manifestó ser o haber sido ciberacosado, siendo mayor el número de mujeres que el de hombres, aunque no estadísticamente significativo, el que se encasilló en este rol. Por otro lado, un $4.5 \%$ de la muestra señaló perpetrar el acoso a través de las TIC. Del total del alumnado envuelto en dinámicas de acoso escolar, son los hombres los más propensos a verse implicados en el rol de bully-acosador (64.52 \%). Asimismo, en lo que respecta al acoso que se ejerce por medio de las TIC fueron, en este caso, las mujeres quienes estaban más relacionadas con este fenómeno, tanto en el rol de víctimas, como en el de acosadorsa (62.07 \% y $70 \%$, respectivamente), en contraposición a los hombres.
Tabla 1

Alumnado enrolado en situaciones de acoso en función del curso

\begin{tabular}{lrrrrrrrr}
\hline \multirow{2}{*}{ Curso } & \multicolumn{1}{c}{ VB } & \multicolumn{1}{c}{ AB } & \multicolumn{2}{c}{ VCB } & \multicolumn{2}{c}{ ACB } \\
\cline { 2 - 9 } & $n=52$ & $\%$ & $n=31$ & $\%$ & $n=29$ & $\%$ & $n=10$ & $\%$ \\
\hline $6 .{ }^{\circ}$ Ed. Primaria & 9 & 22 & & & 5 & 12.2 & - & - \\
$1 .^{\circ}$ de ESO & 26 & 68.4 & 29 & 76.3 & 17 & 44.7 & 9 & 23.7 \\
$2 .^{\circ}$ de ESO & 10 & 16.4 & 1 & 1.6 & 1 & 1.6 & - & - \\
$3 .^{\circ}$ de ESO & 2 & 10.5 & & & 2 & 10.5 & - & - \\
$4 .^{\circ}$ de ESO & 5 & 12.2 & 1 & 2.4 & 4 & 9.8 & 1 & 2.4 \\
\hline
\end{tabular}

Nota. VB: víctimas (acoso); AB: acosadores;

VCB: cibervíctimas; ACB: ciberacosadores.

Atendiendo al curso en el que se hallaba el alumnado de la muestra seleccionada, cabe mencionar que, en alusión al acoso, eran los y las estudiantes de 1.. de ESO, los que, con diferencia, estaban más implicados en este fenómeno, tanto en el rol de víctimas (68.4\%) y, aún más si cabe, como agresores (76.3\%). En cambio, en el alumnado de 1.. de bachillerato no se encontró ningún rastro de acoso en ninguno de los roles.

En lo que respecta al ciberacoso, del mismo modo que ocurre con el acoso, es en el curso de 1. - de ESO donde se hallaron las medias más elevadas tanto en cibervíctimas (44.7\%) como en ciberacosadores $(23.7 \%)$. Coincide también el alumnado de 1.․ de bachillerato, que se encontraba exento de verse inmiscuido en experiencias y/o dinámicas de tal tipo.

A continuación, se exponen las características del acoso y ciberacoso que trataron de dar respuesta a uno de los objetivos propuestos de antemano. Los resultados de frecuencia y porcentaje del interrogante: idesde cuándo se acosaba o se era acosado?, puso de manifiesto que, en referencia al acoso, los porcentajes más altos (32.7\% para las víctimas; $35.5 \%$ para acosadores) se encontraron en la respuesta: menos de un mes. De donde se puede deducir que quizás no estemos en su totalidad ante casos de acoso, sino de agresión. Es reseñable también que el $5.8 \%$ de las víctimas de acoso afirme ser acosado desde el comienzo de su etapa educativa. Caso contrario es el que concierne al ciberacoso, el cual reportó sus porcentajes más altos en más de seis meses (31.03\% para cibervíctimas y $60 \%$ 
para acosadores cibernéticos), lo que caracteriza al acoso como persistente.

Reafirmando lo ya mencionado, los porcentajes que se expresaron en el apartado Frecuencia con que se acosa o se es acosado, revelaron que la mayoría de los ataques de acoso y ciberacoso que se perpetraron o percibieron correspondieron a una frecuencia de 1 o 2 veces al mes (36.54\%), por lo que no alude a la persistencia que caracteriza al acoso.

Cabe señalar por otro lado, que un amplio porcentaje se hallaba expuesto de un modo obstinado, ya que lo sufrían más de tres veces por semana, concretamente un $15.38 \%$ en el caso de las bully-víctimas y un $10.34 \%$, en las víctimas de acoso cibernético. Un total de 23 sujetos no contestaron esta cuestión, por lo que es deducible que en el momento de la recogida de datos no se veían envueltos en tan pernicioso panorama.

A continuación, se hizo referencia a los datos que aportaron los sujetos en relación con la persona o personas que acosaban o por quienes eran agredidos o acosados. En primer lugar, en su mayoría, las víctimas de acoso apuntaron que eran acosadas o agredidas por más de una persona, ya sea un grupo de hombres (26.92 \%), de mujeres (25\%) o hombres y mujeres (11.54\%). Atendiendo a los ciberacosadores, sus víctimas suelen ser en su mayoría hombres $(67.74 \%)$. Respecto a las cibervíctimas, los porcentajes se dividieron de forma más equilibrado, siendo un grupo de mujeres $(24.14 \%)$, en la mayoría de los casos por sujetos de su misma edad (62.07\%), las que reciben el acoso a través de las TIC, medio que hace posible que este acoso no sea necesariamente presencial, de ahí que el 24.14 $\%$ desconozca por quien es acosado. En relación con los acosadores cibernéticos, los hombres (50 \%) son a los que más suelen acosar, siendo, en la mayoría de los casos, además la víctima mayor que él/ella (50\%).
Tabla 2

Formas de acoso

\begin{tabular}{lrrrr}
\hline \multirow{2}{*}{ Formas de acoso } & \multicolumn{2}{c}{ VB } & AB \\
\cline { 2 - 5 } \multicolumn{1}{c}{$n=52$} & \multicolumn{1}{c}{$\%$} & $n=31$ & \multicolumn{1}{c}{$\%$} \\
\hline Ignorar & 5 & 9.6 & 5 & 16.1 \\
Dejar en ridículo & 8 & 15.4 & 4 & 12.9 \\
No querer juntarse & 7 & 13.5 & 3 & 9.7 \\
Insultar o amenazar & 21 & 40.4 & 17 & 54.8 \\
Obligar a hacer cosas que no quiere & 1 & 1.9 & - & - \\
Romper o esconder las cosas & 6 & 11.5 & - & - \\
Pegar & 4 & 7.7 & 2 & 6.5 \\
\hline
\end{tabular}

El modo de acoso más reportado con diferencia por los sujetos que conforman ambos roles fue aquel que se caracteriza por ser de tipo directo verbal, concretamente mediante insultos o amenazas, hallando un $40.4 \%$ por parte de las víctimas y un $54.8 \%$ en el caso de los acosadores. Dejar en ridículo fue la segunda acción más señalada por el alumnado víctima de ciberacoso con un $15.4 \%$. Por otra parte, el acoso físico (pegar) se expresa en un $7.7 \%$ en el rol de víctima y un $6.5 \%$ acosa de dicho modo.

\section{Tabla 3}

Formas de ciberacoso

\begin{tabular}{lcccr}
\hline \multirow{2}{*}{ Formas de ciberacoso } & \multicolumn{2}{c}{ VCB } & \multicolumn{2}{c}{ ACB } \\
\cline { 2 - 5 } & $M$ & $D E$ & \multicolumn{1}{c}{$M$} & \multicolumn{1}{c}{$D E$} \\
\hline Insultar o amenazar & 2.19 & 1.02 & 1.77 & 1.09 \\
Burlarse con imágenes o vídeos & 1.54 & 0.76 & 1.67 & 1 \\
Decir cosas falsas & 1.72 & 0.85 & 1.44 & 1.01 \\
Usar móvil o perfil de usuario para acosar & 1.23 & 0.51 & - & - \\
Contar cosas a otros & 2.27 & 1.12 & 1.67 & 1 \\
\hline
\end{tabular}

$$
\begin{gathered}
\text { Nota. } 1=\text { Nunca } 2=\text { A veces } \\
3=\text { Casi siempre } 4=\text { Siempre }
\end{gathered}
$$

Por otra parte, uno de los tipos de ciberacoso que las víctimas más cuantificaron es el insulto o la amenaza $(M=2.19 ; D E=1.02)$, de igual modo que los ciberacosadores $(M=1.77 ; D E$ $=1.09$ ), coincidiendo este tipo con el señalado por los sujetos enrolados en situaciones de acoso. Contar cosas de las víctimas a otras personas, también fue reportado, especialmente por las cibervíctimas $(M=2.27 ; D E=1.12)$. 
Tabla 4

Vías de ciberacoso

\begin{tabular}{lrrrr}
\hline \multirow{2}{*}{ Vía de ciberacoso } & \multicolumn{2}{c}{ VCB } & \multicolumn{2}{c}{ ACB } \\
\cline { 2 - 5 } & \multicolumn{1}{c}{$M$} & \multicolumn{1}{c}{$D E$} & \multicolumn{1}{c}{$M$} & $D E$ \\
\hline Llamadas & 1.98 & 0.99 & 1.22 & 0.67 \\
WhatsApp & 3.08 & 1 & 2.78 & 0.83 \\
Correos electrónicos & 1.17 & 0.46 & 1.33 & 0.71 \\
Redes sociales & 2.08 & 1.31 & 2.25 & 0.96 \\
Juegos online & 2.4 & 1.07 & 1.75 & 0.96 \\
Páginas web y blogs & 1.2 & 0.42 & 1.75 & 0.96 \\
\hline
\end{tabular}

Nota. 1 = Nunca; 2 = A veces;

$3=$ Casi siempre; $4=$ Siempre

La aplicación móvil Whatsapp se erige como el canal con el que más asiduidad se comete ciberacoso, así fue señalado tanto por las cibervíctimas $(M=3.08 ; D E=1)$ como por los ciberacosadores $(M=2.78 ; D E=0.83)$. No obstante, las redes sociales también fueron bastante señaladas por cibervíctimas $(M=2.08$; $D E=1.31)$ y ciberacosadores $(M=2.25 ; D E=$ 0.96).

\section{Tabla 5}

Comunicación de experiencias de acoso escolar

\begin{tabular}{lrrrr}
\hline \multirow{2}{*}{ Actor } & \multicolumn{3}{c}{ Sí } & \multicolumn{1}{c}{ No } \\
\cline { 2 - 5 } \multicolumn{1}{c}{$n$} & \multicolumn{1}{c}{$\%$} & \multicolumn{1}{c}{$n$} & \multicolumn{1}{c}{$\%$} \\
\hline Víctimas (acoso) & 41 & 78.8 & 11 & 21.2 \\
Agresores (acoso) & 19 & 61.3 & 12 & 38.7 \\
Víctimas (ciberacoso) & 15 & 51.7 & 14 & 48.3 \\
Agresores (ciberacoso) & 7 & 70 & 3 & 30 \\
\hline
\end{tabular}

El conjunto estudiantil víctima de acoso es el que más indicó comunicar lo sucedido, donde de un total de 52 sujetos, un $78.8 \%$ solía contarlo. Atendiendo a las cibervíctimas, solo un poco más de la mitad (51.7\%) comunica los hechos. En comparación, las víctimas señalaron comunicar las experiencias en mayor medida que los agresores, en ambos tipos de acoso.
Análisis de las hipótesis

Tabla 6

Correlaciones bivariadas mediante el coeficiente de Pearson

\begin{tabular}{|c|c|c|c|c|c|c|}
\hline Variable & 1 & 2 & 3 & 4 & 5 & 6 \\
\hline 1. Edad & - & & & & & \\
\hline 2. Sexo & -0.046 & - & & & & \\
\hline 3. Curso & $0.924^{*}$ & -0.017 & - & & & \\
\hline 4. Bully-víctimas & $-0.243^{*}$ & 0.024 & $-0.28^{*}$ & - & & \\
\hline 5. Cibervíctimas & $-0.178^{*}$ & 0.066 & $-0.189^{*}$ & $0.542^{*}$ & - & \\
\hline 6. Bully-acosadores & $-0.249^{*}$ & $-0.147^{*}$ & $-0.269^{*}$ & $0.451^{*}$ & $0.344^{*}$ & - \\
\hline 7. Ciberacosadores & -0.052 & 0.072 & -0.122 & $0.238^{*}$ & $0.367^{*}$ & $0.351^{*}$ \\
\hline
\end{tabular}

Para poner a prueba la $\mathrm{H}_{1}$, se realizó un análisis de correlación bivariada mediante el coeficiente de correlación de Pearson (Tabla 6). Los resultados mostraron una correlación negativa significativa entre la edad del alumnado y la victimización de acoso $\left(r=-0.243^{*}\right)$, corroborándose con una relación significativa del 0.05. Derivándose de la $\mathrm{H}_{1}$, se puso a prueba $\mathrm{H}_{1.1}$, para ello se realizó un ANOVA con la variable independiente curso y la variable dependiente Bully-víctimas.

Tabla 7

ANOVA de un factor, Bully-víctimas - Curso

\begin{tabular}{lrrrrr}
\hline \multicolumn{1}{c}{ ANOVA } & Suma de cuadrados & $\boldsymbol{g l}$ & $\boldsymbol{M}$ & $\boldsymbol{F}$ & $\boldsymbol{p}$ \\
\hline Entre grupos & 9.934 & 5 & 1.987 & 14.279 & 0 \\
Dentro de grupos & 29.775 & 214 & 0.139 & - & - \\
Total & 39.709 & 219 & & - & - \\
\hline
\end{tabular}

En este sentido, los resultados pusieron de manifiesto la presencia de diferencias estadísticamente significativas en función del curso en el que se encuentran, $F_{(5.219)}=14.28$, $p<0.05$. 
Tabla 8

Análisis post hoc (Bonferroni): Bully-víctimas Curso

\begin{tabular}{|c|c|c|c|c|c|}
\hline \multirow{2}{*}{ (I) Curso } & \multirow{2}{*}{ (J) Curso } & \multirow{2}{*}{$\begin{array}{c}\text { Diferencias } \\
\text { de medias } \\
\text { (I-J) }\end{array}$} & \multirow{2}{*}{ Error } & \multicolumn{2}{|c|}{$\begin{array}{l}95 \% \text { de intervalo } \\
\text { de confianza }\end{array}$} \\
\hline & & & & $\begin{array}{l}\text { Límite } \\
\text { inferior }\end{array}$ & $\begin{array}{c}\text { Límite } \\
\text { superior }\end{array}$ \\
\hline \multirow[t]{5}{*}{$1 .^{\circ}$ de ESO } & $6 .^{\circ} \mathrm{EP}$ & $0.4647^{*}$ & 0.08399 & 0.2154 & 0.714 \\
\hline & $2 .^{\circ} \mathrm{de} \mathrm{ESO}$ & $0.52028^{*}$ & 0.07709 & 0.2914 & 0.7491 \\
\hline & $3 .^{\circ} \mathrm{de} \mathrm{ESO}$ & $0.57895^{*}$ & 0.10481 & 0.2678 & 0.8901 \\
\hline & $4 .^{\circ} \mathrm{de}$ ESO & $0.56226^{*}$ & 0.08399 & 0.3129 & 0.8116 \\
\hline & $1 .{ }^{\circ} \mathrm{BCH}$ & $0.68421^{*}$ & 0.10305 & 0.3783 & 0.9901 \\
\hline
\end{tabular}

Realizado el ANOVA, se llevó a cabo un análisis post hoc (Bonferroni) para tratar de comprobar las diferencias existentes entre cada uno de los cursos. De esta manera, los contrastes post hoc indicaron que en los grupos de la variable independiente utilizada se diferencian de forma estadísticamente significativa (1. de ESO y el resto de los cursos $p<0.05)$.

Tabla 9

Descriptivos Bully-acosadores - Sexo

\begin{tabular}{cccrrr}
\hline Grupo & Sexo & $\boldsymbol{n}$ & $\boldsymbol{M}$ & $\boldsymbol{D} \boldsymbol{E}$ & Error \\
\hline \multirow{2}{*}{ Bully-acosadores } & chico & 102 & 1.2 & 0.4 & 0.04 \\
& chica & 118 & 1.09 & 0.29 & 0.03 \\
\hline
\end{tabular}

\section{Tabla 10}

Prueba t Bully-acosadores - Sexo

\begin{tabular}{lcccc}
\hline Bully-acosadores - Sexo & $\boldsymbol{F}$ & $\boldsymbol{t}$ & $\boldsymbol{g l}$ & $\boldsymbol{p}$ \\
\hline Se asumen varianzas iguales & $20.326^{* * *}$ & 2.201 & 218 & 0.029 \\
No se asumen varianzas iguales & & 2.153 & 182.41 & 0.033 \\
\hline
\end{tabular}

$$
* * p<0.001
$$

Para comprobar el efecto que el sexo de la muestra desempeñaba sobre los acosadores de acoso $\left(\mathrm{H}_{2}\right)$, se realizó una prueba $t$ Student para muestras independientes, utilizando la variable independiente sexo, junto con bully-acosadores como variable dependiente. La determinación de los resultados indicó la existencia de diferencias estadísticamente significativas en el grupo bully- acosadores en función del sexo, $t_{(218)}=2.201$, $p=0.029\left(M_{\text {hombres }}=1.2 ; D E=0.4\right.$ versus $\left.M_{\text {mujeres }}=1.09 ; D E=0.29\right)$, confirmando la hipótesis planteada.

\section{Tabla 11}

Descriptivos Cibervíctimas - Bully-víctimas

\begin{tabular}{clrrrr}
\hline Grupo & Bully-víctimas & \multicolumn{1}{c}{$\boldsymbol{n}$} & $\boldsymbol{M}$ & $\boldsymbol{D} \boldsymbol{E}$ & Error \\
\hline \multirow{2}{*}{ Cibervíctimas } & No & 168 & 1.03 & 0.17 & 0.01 \\
& Sí & 52 & 1.46 & 0.5 & 0.07 \\
\hline
\end{tabular}

Tabla 12

Prueba t Cibervíctimas - Bully-víctimas

\begin{tabular}{lcrr}
\hline Cibervíctimas - Bully-víctimas & F & $\boldsymbol{t}$ & \multicolumn{1}{c}{$\boldsymbol{g l}$} \\
\hline Se asumen varianzas iguales & $382.5^{* *}$ & $-9.529^{* *}$ & 218 \\
No se asumen varianzas iguales & & $-6.078^{* *}$ & 54.663 \\
\hline \multicolumn{4}{c}{} \\
$\qquad * p<0.001$.
\end{tabular}

En el análisis de correlación (Tabla 6) efectuado anteriormente, también se denotó una correlación positiva significativa entre la victimización de acoso y la victimización de ciberacoso $(r=0.542)$. En este sentido, para encontrar diferencias en la prevalencia de ambos fenómenos, se llevó a cabo una prueba $t$ Student para muestras independientes con la victimización de acoso como variable independiente, junto con la victimización de ciberacoso como variable dependiente.

Hallados los resultados, se puso de manifiesto un efecto principal de las víctimas de acoso en las víctimas de ciberacoso, puesto que se aprecian puntuaciones más elevadas en la condición de victimización de acoso en los casos de victimización de ciberacoso, $t_{(218)}=-9.529, p<$ $0.05\left(\mathrm{M}_{\mathrm{No}_{\mathrm{o}}}=1.03 ; \mathrm{DE}=0.17\right.$ vs. $\mathrm{M}_{S i}=1.46$; $D E=0.5)$, ratificando de este modo $\mathrm{H}_{3}$.

Dados los hallazgos en $\mathrm{H}_{3}$, se derivó esta hipótesis alternativa, puesto que existían indicios de que ser víctima de acoso podía ser un factor predictivo de que además se fuese ciberacosado. Por tanto, para ver en qué medida el padecer 
acoso predice el hecho de sufrir ciberacoso se calculó una regresión lineal simple.

\section{Tabla 13}

Regresión lineal simple de Cibervíctimas-Bullyvíctimas

\begin{tabular}{cccc}
\hline Grupo & $\boldsymbol{\beta}$ & $\boldsymbol{t}$ & $\boldsymbol{p}$ \\
\hline Cibervíctimas - Bully-víctimas & 0.542 & 9.529 & 0 \\
\hline
\end{tabular}

Una vez concluidos los resultados, cabe decir que estos apuntaron a la victimización de acoso como variable predictiva de la victimización de ciberacoso $(\beta=0.542, p<0.05)$, de modo que en este caso se confirma que una mayor victimización de acoso predice una mayor victimización de ciberacoso.

\section{Discusión}

En relación con el primero de los objetivos planteados en este trabajo, Identificar la prevalencia de acoso y ciberacoso entre adolescentes, se observa un alto nivel de prevalencia en comparación con otros estudios (Garaigordobil, 2011; Larrain \& Garaigordobil, 2020), tanto en la victimización de acoso como en los acosadores. Así mismo, en relación con las víctimas, un porcentaje significativo lo han padecido un máximo de hasta dos veces al mes, encontrándose estos resultados por debajo de los aportados (47.2\%) por Nacimiento y Mora-Merchán (2014). Estos datos hacen notoria la necesidad de poner en marcha estrategias urgentes de intervención en los centros educativos (Tesouro et al., 2005), dado que el aumento del porcentaje es alarmante.

En relación con el segundo de los objetivos propuestos, Conocer las características de acoso y ciberacoso en función del rol (víctima/ agresor), y en la línea de que lo que señala Calamestra (2014), se puede observar que las víctimas señalaron en su mayoría ser intimidadas por grupos de hombres, y el tipo verbal es el más frecuente por lo cual son afectados rotundamente, encuadrando este tipo de acoso como directo.

Por otra parte, la gran mayoría del alumnado que es víctima de acoso tradicional, la comunicaba lo sucedido a otras personas. Pudiéndose entender esto como una estrategia de afrontamiento ante el acoso, tal y como recogen otros trabajos como el de Waasdorp y Bradshaw (2015) o el de Cava (2011).

Es relevante que el porcentaje encontrado de víctimas de ciberacoso es bastante superior al mencionados en la mayoría de los estudios tomados como referencia (Arnaiz et al., 2016; Ortega et al., 2018; Polo et al., 2014). Quedando únicamente por debajo de los datos aportados por el estudio de Kubiszewski et al. (2015) que llegaban a un $18 \%$. Así mismo, en relación con los acosadores cibernéticos, el porcentaje se reduce al $4.5 \%$, encontrándose en la línea de los trabajos de Kubiszewski et al. (2015) y Bartrina (2014), pero superior al reportado por Arnaiz et al. (2016) que se encuentra en un 1.5 $\%$ de los sujetos. Siguiendo la idea de Giménez et al. (2015), se encontró que la principal vía de ciberacoso es a través del smartphone, concretamente, tanto los acosadores como las víctimas señalan la aplicación Whatsapp como el medio más utilizado para ello. Para terminar con este segundo objetivo, cabe señalar que la edad en la que más casos de estudiantado víctima se encontró fue de 12 años, coincidiendo con el intervalo sugerido por Piñuel y Oñate (2007), siendo por tanto, el alumnado de 1. de ESO el más implicado en el ámbito del acoso escolar.

En relación con las hipótesis que se plantearon en este trabajo, $\mathrm{H}_{1}$ y $\mathrm{H}_{1.1}$, referidas a que existe una menor prevalencia de acoso en la medida que el alumnado aumenta la edad, y dado que se han encontrado diferencias significativas entre los cursos, podemos concluir que estas hipótesis se confirman, observando diferencias significativas no solo en 1. $\mathrm{o}$ de ESO, como era de esperar, sino en el resto de los cursos. Este resultado se acerca a lo sugerido por Smith (2012), el cual sitúa el último curso de educación primaria como pico de la intimidación, lo cual hace recomendable establecer pautas directas de actuación en esta 
etapa educativa, más aún cuando se trata de un momento cercano al cambio de ciclo.

Atendiendo a la segunda hipótesis, La mayor implicación en acoso por parte de los alumnos que de las alumnas, hay que mencionar que también se acepta aunque se debe ser cauteloso ya que, aunque trabajos como el de GarcíaFernández (2013), o más recientemente el Aranda et al. (2015), avalan una mayor participación de los hombres en el acoso tradicional, sobre todo en el rol de agresor, se matiza en el reciente estudio de Chocarro y Garaigordobil (2019), en el que se observa una frecuencia significativamente mayor en conductas de agresión física que las mujeres, no encontrándose diferencias significativas en la realización de conductas de agresión social y psicológica, lo cual parece indicar que no deben crearse propuestas de intervención diferenciadas por sexo.

En las $\mathrm{H}_{3}$ y $\mathrm{H}_{3.1}$, sobre la existencia de diferencias en la prevalencia de ciberacoso en función de si previamente se ha padecido acoso, y el hecho de que sufrir acoso tradicional sea predictor de sufrir ciberacoso, cabe destacar que ambas se ratificaron. Los datos que se hallaron son afines a los expuestos por Hinduja y Patchim (2010), los cuales reportaron que el $65 \%$ de las cibervíctimas de su investigación, también había adoptado el mismo rol en el acoso tradicional, por lo que no parece que exista un patrón específico de ciberacosadores.

Como conclusión final, entendemos que, a la luz de los datos obtenidos, para la prevención e intervención del acoso y el ciberacoso, es fundamental que tanto los docentes como las familias, posean estrategias de registro, observación y delimitación de los casos específicos de acoso entre pares que puedan producirse tanto en el ámbito educativo, labor de los docentes, como en el familiar mediante el ciberacoso. Dada la complejidad de este fenómeno, se requieren acciones igualmente complejas y estructuradas que estén ajustadas tanto al contexto físico como como al temporal. Es en este sentido en el que se recomienda una formación específica de los docentes para poder recoger posibles acciones de acoso y poder realizar intervenciones específicas mediante dinámicas de socialización.

Para concluir, se considera conveniente el trazar unas futuras líneas de trabajo que inviten a continuar determinando las características de este fenómeno tan actual. Es esta actualidad la que lo provee como un tema de estudio atractivo a la par que relevante, debido a que los principales afectados se hallan aunados en el sector juvenil de nuestra población. Por estos motivos, resultaría interesante llevar a cabo el mismo estudio o uno de características similares, aumentando la muestra e incluso considerando a otras poblaciones que, dado el caso, también puedan verse afligidas por ambas manifestaciones de acoso.

Asimismo, está en proyecto realizar y validar un cuestionario de diseño propio con el que se pueda profundizar en lo concerniente al acoso cibernético, incluyendo nuevas variables, sobre todo de aquellas que puedan llegar a relacionarlo con el contexto familiar y la personalidad de los sujetos, actualizando algunos canales de acoso.

\section{Referencias}

Álvarez-García, D., Núñez, J. C., Álvarez, L., Dobarro, A., Rodríguez, C., \& GonzálezCastro, P. (2011). Violencia a través de las tecnologías de la información y la comunicación en estudiantes de secundaria. Anales de Psicología, 27(1), 221-231. https://www.redalyc.org/pdf/167/ 16717018026.pdf

Aranda, M., Montes-Berges, B., \& CastilloMayén, M. R. (2015). La orientación a la dominancia social en la adolescencia: el papel de la identidad de género y los rasgos estereotípicos femeninos y masculinos. Revista de Psicología Social, 30(2), 241-263. https://doi.org/10.1080/21 711976.2015 .1016752

Arnaiz, P., Cerezo, F., Giménez, A. M., \& Maquilón, J. J. (2016). Conductas de ciberadicción y experiencias de ciberacoso entre adolescentes. Anales de Psicología, 
32(3), 761-769. https://doi.org/10.6018/an alesps.32.3.217461

Bartrina, M. J. (2014). Conductas de ciberacoso en niños y adolescentes. Hay una salida con la educación y la conciencia social. Educar, 50(2), 386-400. https://doi.org/10.5565/rev /educar.672

Calmaestra, J. (2011). Ciberacoso: prevalencia y características de un nuevo tipo de acoso indirecto (Tesis doctoral inédita). Universidad de Córdoba, España.

Campbell, M., Spears, B., Slee, P., Butler, D., \& Kift, S. (2012). Victims' perceptions of traditional and cyberbullying, and the psychosocial correlates of their victimisation. Emotional and Behavioural Difficulties, 17(3-4), 389-401. https://doi.or g/10.1080/13632752.2012.704316.

Cañón-Rodríguez, R. (2018). Ciberacoso: revisión de la literatura educativa en español. Revista Latinoamericana de Tecnología Educativa, 17(2), 87-99. https://d oi.org/10.17398/1695-288X.17.2.87

Castan\#eda-Va\#zquez, C., Moreno-Arrebola, R., Gonza\#lez-Valero, G., VicianaGaro\#fano, V., \& Zurita-Ortega, F. (2020). Posibles relaciones entre el acoso y la actividad fi\#sica: una revisio\#n sistema\#tica. Journal of Sport and Health Research, 12(1), 94-111. http://hdl.handle. net/10481/58901

Cava, M. J. (2011). Familia, profesorado e iguales: claves para el apoyo a las víctimas de acoso escolar. Psychosocial Intervention, 20(2), 183-192. https://doi.org/10.5093/in2 011 v20n2a6

Cerezo, F., \& Alto, M. (2010). Social status, gender, classroom climate and bullying among adolescents' pupils. Anales de Psicología, 26(1), 137-144. https://doi.org/1 $0.6018 / 92131$

Chocarro, E., \& Garaigordobil, M. (2019). Bullying y cyberbullying: diferencias de sexo en víctimas, agresores y observadores. Pensamiento Psicolo\#gico, 17(2), 57-71. https://doi.org/10.11144/Jave rianacali.PPSI17-2.bcds
Colás, P. (1994). Los métodos descriptivos. En P. Colás \& L. Buendía (Eds.), Investigación educativa (2. ed.) pp. 177-200. Alfar.

De la Caba, M. A., \& López, R. (2013). La agresión entre iguales en la era digital: estrategias de afrontamiento de los estudiantes del último ciclo de primaria y del primero de secundaria. Revista de Educación, 362, 247-272. https://doi.org/10 .4438/1988-592X-RE-2011-362-160

Estévez, A., Villardón, L., Calvete, E., Padilla, P., \& Orue, I. (2010). Adolescentes víctimas de ciberacoso: prevalencia y características. Psicología Conductual, 18(1), 73-89. https://www.behavioralpsycho.com/ wp-content/uploads/2019/08/04.Estevez_1 8-1r.pdf

Fitzpatrick, K. M., Dulin, A. J., \& Piko, B. F. (2007). Not just pushing and shoving: School bullying among African American adolescents. Journal of School Health, 77(1), 16-22. https://doi.org/10.1111/j.1746-1561 .2007.00157.x

Francisco, S. M., Simao, A. M. V., Ferreira, P. C., \& das Dores Martins, M. J. (2015). Cyberbullying: The hidden side of college students. Computers in Human Behavior, 43, 167-182. https://doi.org/10.1016/j.chb.201 4.10.045.

Garaigordobil, M. (2011). Prevalencia y consecuencias del ciberacoso: una revisión. International Journal of Psychology and Psychological Therapy, 11(2), 233-254. https://www.redalyc.org/pdf/560/ 56019292003.pdf

García-Fernández, J. M., Martínez-Monteagudo, M. C., \& Inglés, C. J. (2013). ¿Cómo se relaciona la ansiedad escolar con el rendimiento académico? Revista Iberoamericada de Psicología y Salud, 4(1), 63-76. https://www.redalyc.org/pdf/2451/2 45126428003.pdf

Gázquez, J. J., Pérez-Fuentes, M. C., Carrión, J. J., \& Santiuste, V. (2010). Estudio y análisis de conductas violentas en educación secundaria en España. Universitas Psychologica, 9(2), 
371-380. https://doi.org/10.11144/Javerian a.upsy9-2.eacv

Giménez, A. M., Maquilón, J. J., \& Arnaiz, P. (2014). Acceso a las tecnologías, rendimiento y ciberacoso en escolares de educación secundaria. Revista Iberoamericana de Psicología y Salud, 5(2), 119-133. https://www.redalyc.org/pdf/2451 /245131498002.pdf

Giménez, A. M., Maquilón, J. J., \& Arnaiz, P. (2015). Usos problemáticos y agresivos de las TIC por parte de adolescentes implicados en ciberacoso. Revista de Investigación Educativa, 33(2), 335-351. htt ps://doi.org/10.6018/rie.33.2.199841

Gómez, J., \& Hidalgo, M. (2002). La validez en los test, escalas y cuestionarios. Universidad de Antioquia. Centro de Estudios de Opinión.

Herrera-López, M., Romera, E., \& Ortega-Ruiz, R. (2018) Acoso y ciberacoso en Latinoamérica. Un estudio bibliométrico. Revista Mexicana de Investigación Educativa, 23(76), 125-155. http://www.scielo.org.mx/scielo.p hp?script $=$ sci_arttext\&pid $=$ S1405-66662 $01800010012 \overline{5}$

Hinduja, S., \& Patchin, J. W. (2010). Bullying, cyberbullying, and suicide. Archives of Suicide Research, 14(3), 206-221. https://do i.org/10.1080/13811118.2010.494133

Juvonen, J., \& Gross, E. F. (2008). Extending the school grounds? Bullying experiences in ciberspace. Journal of School Health, 78, 496-505. https://doi.org/10.1111/j.1746-15 61.2008.00335.x

Kowalski, R. M., \& Limber, S. P. (2007). Electronic bullying among middle school students. Journal of Adolescent Health, 41(6), 22-30. https://doi.org/10.1016/j.jado health.2007.08.01

Kubiszewski, V., Fontaine, R., Potard, C., \& Auzoult, L. (2015). Does cyberbullying overlap with school bullying when taking modality of involvement into account? Computers in Human Behavior, 43, 49-57. h ttps://doi.org/10.1016/j.chb.2014.10.049

Larrain, E., \& Garaigordobil, M. (2020). El bullying en el País Vasco: prevalencia y diferencias en función del sexo y la orientación sexual. Clínica y Salud, 31(3), 147-153. https://doi.org/10.5093/clysa2020 a19

López, V., Bilbao, M., \& Rodríguez, J. I. (2012). La sala de clases sí importa: incidencia del clima de aula sobre la percepción de intimidación y victimización entre escolares. Universitas Psychologica, 11(1), 91-101. https://doi.org/10.11144/Javeriana. upsy11-1.scii

Messick, S. (1989). Validity. En R. Linn (Ed.), Educational measurement (3.. ed., pp 13-103). Macmillan.

Modecki, K. L., Minchin, J., Harbaugh, A. G., Guerra, N. G., \& Runions, K. C. (2014). Bullying prevalence across contexts: A meta-analisys measuring ciber and traditional bullying. Journal of Adolescent Health, 55(5), 602-611. https://d oi.org/10.1016/j.jadohealth.2014.06.007

Nacimiento, L., \& Mora-Merchán, J. A. (2014). El uso de estrategias de afrontamiento y habilidades metacognitivas ante situaciones de acoso y ciberacoso. European Journal of Education and Psychology, 7(2), 121-129. https://doi.org/1 0.1989/ejep.v7i2.184

Naylor, P., Cowie, H., \& Del Rey, R. (2001). Coping strategies of secondary school children in response to being bullied. Child Psychology E Psychiatry, 6(3), 114-120. http s://doi.org/10.1017/S1360641701002647

Olweus, D. (1993). Bullying at school: What we know and what we can do. Blackwell.

Olweus, D. (2013). School bullying: development and some important challenges. Annual Review of Clinical Psychology, 9, 751-780. https://doi.org/10.1 146/annurev-clinpsu-050212-185516

Ortega, R., Calmaestra, J., \& Mora-Merchán, J. (2008). Cyberbullying. International Journal of Psychology and Psychological Therapy, 8(2), 183-192. https://www.redalyc.org/pdf/560/ 56080204.pdf

Ortega, R., \& Monks, C. P. (2007). Violencia escolar y bullying. Informació Psicològica, 87, 29-37. http://www.informaciopsicologica.in 
fo/OJSmottif/index.php/leonardo/article/vi ew/311/264

Piñuel, I., \& Oñate, A. (2007). Acoso y violencia escolar en España. Informe Cisneros $\chi$. Instituto de Innovación Educativa y Desarrollo Directivo.

Polo, M. I., León, B., Felipe, E., \& Gómez, T. (2014). Ciberacoso en tercer ciclo de educación primaria: variables moduladoras y consecuencias sobre la ansiedad. Apuntes de Psicología, 32(1), 5-14. https://core.ac.uk /download/pdf/190375491.pdf

Polo, M. I., Mendo, S., Fajardo, F., \& León, B. (2017). Una intervención en aprendizaje cooperativo sobre el perfil del observador en la dinámica acoso. Universitas Psychologica, 16(1), 1-13. https://doi.org/10.11144/Javeri ana.upsy16-1.iacp

Powell, M. D., \& Ladd, L. D. (2010). Bullying: A review of the literature and implications for family therapists. American Journal of Family Therapy, 38(3), 189-206. https://doi.org/10 $.1080 / 01926180902961662$

Scheithauer, H. (2002). Unprototypical forms of aggression among children: A meta-analysis. Trabajo presentado en el Workshop on Aggression, Vienna, Austria.

Schneider, S. K., O’Donnell, L., Stueve, A., \& Coulter, R. W. S. (2012). Cyberbullying, school bullying, and psychological distress: A regional census of high school students. American Journal of Public Health, 102, 171-177. https://doi.org/10.2105/AJPH.20 11.300308

Slonje, R., Smith, P. K., \& Frisén, A. (2012). Processes of cyberbullying, and feelings of remorse by bullies: A pilot study. European Journal of Developmental Psychology, 9, 244-259. https://doi.org/10.1080/17405629 .2011 .643670

Slonje, R., Smith, P. K., \& Frisén, A. (2013). The nature of ciberbulliyng, and strategies for prevention. Computers in Human Behavior, 29(1), 26-32. https://doi.org/10.1016/j.chb. 2012.05.024

Smith, P. K. (2012). Cyberbullying and ciber aggression. En S. R. Jimerson, A. B. Nickerson, M. J. Mayer \& M. J. Furlog
(Eds.), Handbook of school violence and school safety: International research and practice (pp. 93-103). Rooutledge.

Tesouro, M., Gu\#ell, M., Moreno, J., \& Franco, M. (2005). Transformemos la educación y mejoremos la escuela trabajando con un programa de competencia social en el aula. REICE. Revista Electrónica Iberoamericana sobre Calidad, Eficacia y Cambio en Educación, 3(1), 487-493. https://www.reda lyc.org/pdf/551/55130148.pdf

Tokunaga, R. S. (2010). Following you home from school: A critical review and synthesis of research on cyberbullying victimization. Computers in Human Behavior, 26, 227-287. https://doi.org/10.1016/j.chb.2009.11.014

Waasdorp, T. E., \& Bradshaw, C. P. (2015). The overlap between cyberbullying and traditional bullying. Journal of Adolescent Health, 56(5), 483-488. https://doi.org/10.1 016/j.jadohealth.2014.12.002

\section{Notas}

* Artículo de investigación. 